\title{
Efficacy of an Intranasal Tear Neurostimulator in Sjögren Syndrome Patients
}

\author{
Jonathan Lilley' \\ Erin C O’Neil' \\ Vatinee $Y$ Bunya' \\ Kennedy Johnson' \\ Gui-Shuang Ying ${ }^{2}$ \\ Peiying Hua ${ }^{2}$ \\ Mina Massaro-Giordano' \\ 'Department of Ophthalmology, Scheie \\ Eye Institute, Perelman School of \\ Medicine at the University of \\ Pennsylvania, Philadelphia, PA, USA; \\ ${ }^{2}$ Center for Preventive Ophthalmology \\ and Biostatistics, University of \\ Pennsylvania, Philadelphia, PA, USA
}

Correspondence: Mina Massaro-Giordano Department of Ophthalmology, Scheie Eye Institute, Perelman School of Medicine at the University of Pennsylvania, 5I N. 39th

Street, Philadelphia, PA, 19104, USA

Tel +I 215-662-9393

Email mina@pennmedicine.upenn.edu
Purpose: To assess the efficacy and safety of an intranasal tear neurostimulator (ITN) device in Sjögren syndrome (SS) patients.

Methods: This was a two-visit prospective, randomized, controlled, same-day crossover study in participants with SS. Inclusion criteria were assessed at a baseline screening visit and included an Ocular Surface Disease Index (OSDI) score $\geq 13$, and a Schirmer with anesthesia $\leq 10 \mathrm{~mm} / 5 \mathrm{~min}$ (in at least one eye), with a cotton swab stimulation induced increase of $\geq 4 \mathrm{~mm}$ in the same eye. Participants returned for the application visit, where they received intranasal and extranasal applications of the ITN in random sequence, separated by at least $60 \mathrm{~min}$. Schirmer scores were measured in both eyes after each application and compared to baseline values. Generalized linear models were performed to compare the change in Schirmer scores from baseline, and generalized estimating equations were used to account for correlations from repeated measurements in the same eye and measurements from two eyes of the same patient.

Results: Fifty-five participants were screened and 35 were enrolled (all female), ranging in age from 31 to 72 years (mean, 57 years). The baseline OSDI score ranged from 14 to 91 (mean, 50.5), and the baseline Schirmer score had a mean (SD) of 6.4 (3.5) ranging from 0 to $20(\mathrm{~mm} / 5 \mathrm{~min})$. Improvement in Schirmer scores was significantly greater for intranasal device application $(13.5 \mathrm{~mm} / 5 \mathrm{~min}, 95 \% \mathrm{CI}$ : 10.4, 16.5) compared to extranasal device application $(0.8 \mathrm{~mm} / 5 \mathrm{~min}, 95 \% \mathrm{CI}:-0.9,2.4)(\mathrm{p}<0.0001)$. The effects of the intranasal device application were significant regardless of the participant's baseline Schirmer score and systemic SS medication usage $(\mathrm{p}<0.05)$.

Conclusion: Intranasal application of the ITN device significantly increased tear production in a subset of SS patients compared to baseline and was more effective than extranasal application. While production of the ITN device was recently discontinued, our findings suggest that other therapies that neurostimulate the lacrimal function unit may be effective in a subset of SS patients.

Keywords: Sjögren syndrome, intranasal tear neurostimulation, tear production

\section{Introduction}

Sjögren syndrome (SS) is an autoimmune disease that affects the mucous membranes and moisture producing glands in the body. It can impact multiple organs throughout the body, resulting in joint pain and fatigue, but two of the most common symptoms are dry eyes and dry mouth. ${ }^{1} \mathrm{SS}$ impacts nearly four million Americans, with the majority being female and an estimated prevalence of 2.2 to 10.3 per 10,000 people. $^{2-5}$ While there are a variety of therapies available to treat the symptoms of SS, none are effective in all patients and additional treatments are needed. ${ }^{6}$ 
In 2017, an intranasal tear neurostimulator (ITN) device was approved by the Food and Drug Administration (FDA) for the treatment of dry eye disease. The ITN stimulates the nerves supplying the Lacrimal Functional Unit (LFU) to temporarily increase tear production. The LFU is comprised of the lacrimal glands and the ocular surface, including the conjunctiva, cornea, and goblet cells, as well as the sensory and motor nerves that connect these structures to maintain tear production and quality. ${ }^{7}$ The afferent pathway of this system includes the ophthalmic and maxillary branches of the trigeminal nerve, with abundant nerve fiber endings in the nasal mucosa. Normally, the trigeminal nerve is responsible for sensing pain and irritation in the nasal cavity and works to expel any foreign body or irritant that enters into the nasal cavity by prompting tear secretion. ${ }^{8}$ The ITN stimulates the afferent nerves of the trigeminal nerve in the nasal cavity, which send an electrical signal to the lacrimal center in the brain. The lacrimal center then sends out electrical signals via efferent autonomic secretomotor nerves, of the seventh cranial nerve (facial nerve), which stimulates the lacrimal glands and tear production. ${ }^{8,9}$

Previous clinical trials have demonstrated that the ITN is effective at stimulating tear production. ${ }^{6,7,10}$ For example, Friedman et al found that stimulation with the ITN significantly increased Schirmer scores and decreased ocular staining and symptom scores from baseline. ${ }^{10}$ In addition, Cohn et al found that the ITN was effective in inducing acute tear production after 90 days of use. ${ }^{11}$ Sheppard et al also found that the ITN was effective in stimulating tear production with acute and long-term use. ${ }^{7}$ There is also evidence that in addition to stimulating the lacrimal glands, the ITN may also stimulate the production of other components of the tear film including mucin and lipid. ${ }^{12,13}$

While there is growing evidence that the ITN is effective at stimulating various components of the tear film, previous studies only included a few SS patients. As a result, evidence is lacking regarding the efficacy of the device in this subset of dry eye patients. Therefore, we set out to study the efficacy and safety of the ITN in a small cohort of SS patients.

\section{Methods}

This was a prospective, randomized, patient-masked, controlled crossover study performed in participants with SS. The trial, conducted in accordance with the principles of the Declaration of Helsinki, was approved by the
Institutional Review Board of the University of Pennsylvania, and was performed in compliance with all applicable laws. It was registered on ClinicalTrials.gov (NCT03719885), and informed consent was obtained. The study was conducted from November 2018 through April 2019.

We will share de-identified participant data that underlie the results reported in this manuscript for a period of 36 months following article publication. Data will be shared with researchers who provide a methodologically sound proposal to achieve the aims in the approved proposal. Proposals should be directed to the corresponding author, Dr Mina Massaro-Giordano, at mina@pennmedicine. upenn.edu. To gain access, data requestors will need to sign a data access agreement.

\section{Subjects}

Eligible participants were greater than 21 years old and had a clinical diagnosis of SS as defined by the AmericanEuropean Consensus Group (AECG), American College of Rheumatology (ACR), or American College of Rheumatology/European League Against Rheumatism (ACR/EULAR) classification criteria. ${ }^{14}$ Participants were also required to have an Ocular Surface Disease Index ${ }^{\circledR}$ (OSDI) total score $\geq 13$ at the Baseline Visit, and they had to demonstrate that cotton swab stimulation induced an increase of $\geq 4 \mathrm{~mm} / 5 \mathrm{~min}$ of the Schirmer with anesthesia score in the same eye. Additional inclusion criteria regarding the Baseline Visit exam are detailed below in the "Study Design" section. Exclusion criteria included the use of any topical eye drops (including artificial tears) within 4 hours of any study visit, history of corneal transplant in either eye, chronic or recurrent epistaxis, systemic anticoagulant use, and coagulation disorders or other conditions that, in the opinion of the investigator, may lead to clinically significant increased bleeding. Additional exclusion criteria included a history of nasal or sinus surgery, history of significant nasal trauma, severely deviated septum, implanted electronic devices including defibrillators or pacemakers, known hypersensitivity to any study agents or materials, and either being pregnant or nursing at the time of any study visit.

\section{Study Design}

Eligibility was determined during the baseline visit. In order to qualify, participants need to have a Schirmer with anesthesia score of $\leq 10 \mathrm{~mm} / 5 \mathrm{~min}$ in at least 1 eye. Participants were then provided with sterile cotton swabs 
and instructed to swirl the swabs in their nasal cavity for 5 min. If the cotton swab stimulation produced an increased Schirmer with anesthesia score of $\geq 4 \mathrm{~mm}$ in the qualifying eye(s), participants were deemed eligible. Enrolled participants then returned within 45 days for the application visit. During the visit, they received two test applications (intranasal and extranasal) in random sequence (Figure 1), separated by at least $60 \mathrm{~min}$. Participants were randomized using an online randomizer and were trained by qualified study personnel on the proper use of the ITN for the intranasal and extranasal applications. For the intranasal application, briefly, the device was turned on and the tips of the device were inserted into the nasal cavity for approximately $3 \mathrm{~min}$ (Figure 1B). For the extranasal application, the device was turned on and the tips were placed on the outside surface of the nose, just below the nasal bridge, for approximately $3 \mathrm{~min}$ (Figure 1C). During each randomized application, a Schirmer test with anesthesia was performed to measure the stimulated tear production.

\section{Statistical Methods}

We calculated the change from baseline in Schirmer score (primary outcome) after each stimulation. Data from both eyes of each patient was included in the statistical analysis and the inter-eye correlation was accounted for by using a generalized estimating equation. We used generalized linear regression models for evaluating the effect of order of intranasal and extranasal device application on change of Schirmer score from baseline, and to compare change of Schirmer score from baseline between the two stimulation methods. A generalized estimating equation (GEE) was used to account for correlations in Schirmer scores from the two stimulation methods in the same eye and between two eyes of the same patient. Subgroup analyses for these comparisons were performed by potential indicators of SS disease severity, which included systemic SS medications status (yes/no), and by baseline Schirmer test result $(<5 \mathrm{~mm}$ vs $\geq 5 \mathrm{~mm})$. All statistical comparisons were performed in SAS v9.4, and two-sided $\mathrm{p}<0.05$ was considered to be statistically significant. For this pilot trial, no formal power sample size or power calculation was performed.

\section{Results}

Fifty-five participants were screened and 35 eligible SS patients were enrolled. All participants were female, and 26 (74\%) were Caucasian (Table 1). Participants ranged in age from 31 to 72 years (mean, 57; $\mathrm{SD}=11.4$ ) and the mean baseline OSDI score was 50.5 (range, 14-91; $\mathrm{SD}=21.0)$. Among the 35 patients, 26 (74.3\%) were taking SS medication at baseline. Schirmer score was measured at baseline in both eyes of each patient and the mean was $6.4 \mathrm{~mm} / 5 \mathrm{~min}(\mathrm{SD}=3.5)$ ranging from 0 to $20 \mathrm{~mm} / 5 \mathrm{~min}$.

Improvement in Schirmer scores from baseline was significantly greater for intranasal device application $(13.5 \mathrm{~mm} /$ $5 \mathrm{~min}, 95 \% \mathrm{CI}: 10.4,16.5 \mathrm{~mm} / 5 \mathrm{~min}$ ) compared to extranasal device application $(0.8 \mathrm{~mm} / 5 \mathrm{~min}, 95 \% \mathrm{CI}:-0.9,2.4 \mathrm{~mm} /$ $5 \mathrm{~min})(\mathrm{p}<0.0001)$ (Table 2$)$. The order of stimulation did not impact the results for intranasal stimulation with a mean change from baseline in Schirmer test score of $12.7 \mathrm{~mm} /$ 5 min when external stimulation was performed first and $13.9 \mathrm{~mm} / 5 \mathrm{~min}$ when internal stimulation was performed first $(p=0.68)$. Similarly, the order did not impact the results of extranasal stimulation with a mean change from baseline in Schirmer test score of $1.13 \mathrm{~mm} / 5 \mathrm{~min}$ when external stimulation was performed first and $0.59 \mathrm{~mm} / 5 \mathrm{~min}$ when internal stimulation was performed first $(\mathrm{p}=0.75)$.

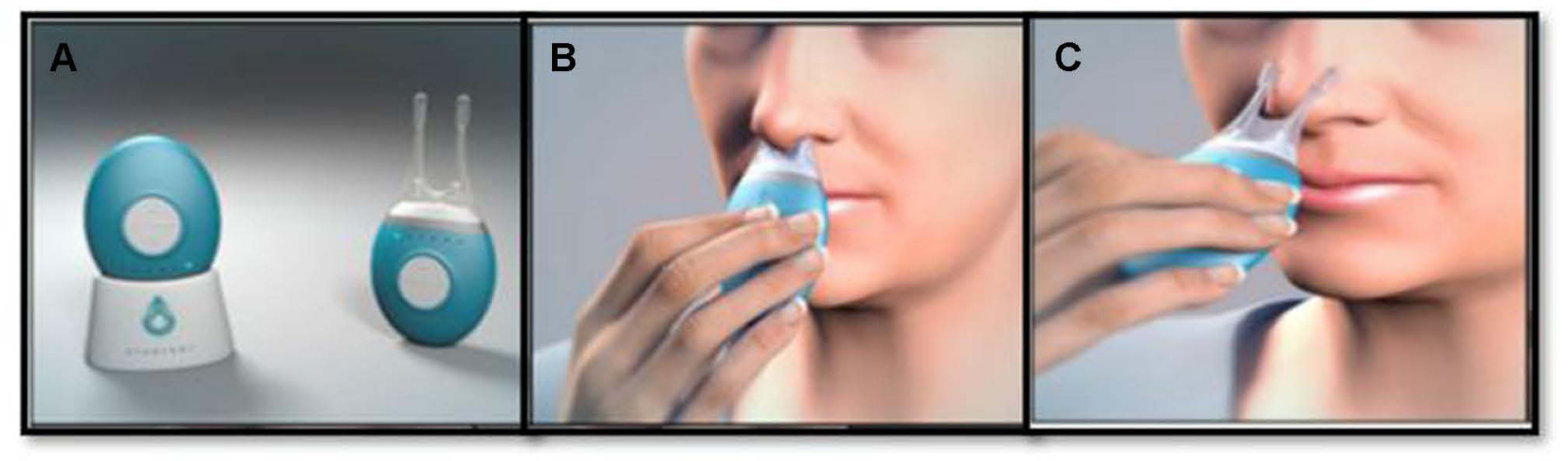

Figure I Intranasal neurostimulator and various application methods. (A) Intranasal neurostimulator device; (B) intranasal application; (C) extranasal application (Photos courtesy of Allergan(C). 
Table I Demographic and Clinical Characteristics of Patients at Baseline $(n=35)$

\begin{tabular}{|l|c|}
\hline Baseline Characteristics & \\
\hline Age (years): Mean (SD) & 57 (I I.4) \\
\hline Race, $n$ (\%) & $26(74)$ \\
White & $5(14)$ \\
Black & $2(6)$ \\
Asian & $2(6)$ \\
Unknown & \\
\hline Ethnicity, $n$ (\%) & $1(3)$ \\
Hispanic or Latino & $34(97)$ \\
\hline Non-Hispanic or Latino & $50.5(21.0)$ \\
\hline Ocular Surface Disease Index Score: Mean (SD) & $6.4(3.5)$ \\
\hline Schirmer score (mm/5min): Mean (SD) & $53(75.7)$ \\
Schirmer <5, eyes (\%) & \\
Schirmer $\geq 5$, eyes (\%) & $26(74.3)$ \\
\hline On systemic medications for Sjögren syndrome, $n$ (\%) \\
Yes & $9(25.7)$ \\
\hline No
\end{tabular}

The improvement using the intranasal device application compared to the extranasal application was found to be significantly greater for all subgroups $(p<0.007)$ (Table 2). When comparing the intranasal device application improvement between subgroups, neither the participant's baseline Schirmer value grouping $(p=0.51)$ nor systemic SS medication usage $(p=0.30)$ had a significant impact on the improvement in Schirmer score (Table 3).

There was one non-serious adverse event occurred among all participants. One participant had a small subconjunctival hemorrhage that resolved a week later without any intervention.

\section{Discussion}

We found that intranasal application of the ITN device significantly increased tear production in a subset of SS patients. Previous studies have evaluated the efficacy of the ITN device in dry eye participants, but no prior studies have exclusively examined SS patients. ${ }^{6,7,10}$ For example, two pivotal studies by Sheppard et al found that the ITN was effective in stimulating tear production with acute and long-term use. ${ }^{7}$ The first study was designed as a singleday crossover study in which 48 participants with dry eye received three consecutive ITN applications at a single visit (intranasal, extranasal, and sham/inactive intranasal). They found that intranasal application of the ITN significantly increased tear production more than extranasal or sham application. In the second study, 89 dry eye patients were instructed to use the ITN device 2-10 times per day for 180 days. The authors found that intranasal application of the ITN significantly increased Schirmer scores by an average of $9.4 \mathrm{~mm}$ as compared to unstimulated scores at 180 days. For comparison, SS patients in our study had an average increase of $13.5 \mathrm{~mm}$ compared to their unstimulated baseline.

Later Pattar et al studied 143 dry eye patients and examined symptom relief after a single application and after multiple, daily use (greater than two times daily) of the device for 45 days utilizing a controlled adverse environment. ${ }^{6}$ The authors found that after a single intranasal application of the device, participants experienced a statistically significant average increase of $16.5 \mathrm{~mm}$ in tear production relative to baseline. In addition, after 45 days of daily use, participants experienced an average increase of $15.2 \mathrm{~mm}$, which was statistically significant. The improvement in tear production also correlated with

Table 2 Comparison Between the Effect of Extranasal and Intranasal Stimulation on Change from Baseline in Schirmer Test Scores in All Participants and by Subgroups

\begin{tabular}{|c|c|c|c|c|c|}
\hline \multirow[b]{2}{*}{$\begin{array}{l}\text { Change from baseline in Schirmer test } \\
\text { score }\end{array}$} & \multirow[b]{2}{*}{$\begin{array}{l}\text { All participants }(n=70 \\
\text { eyes) }\end{array}$} & \multicolumn{2}{|c|}{ Sjögren Syndrome Medication Use } & \multicolumn{2}{|c|}{ Baseline Schirmer Score $(\mathrm{mm} / 5 \mathrm{~min})$} \\
\hline & & $\begin{array}{l}\text { Yes } \\
(n=52 \text { eyes })\end{array}$ & $\begin{array}{l}\text { No } \\
\text { ( } n=18 \text { eyes) }\end{array}$ & $\begin{array}{l}<5 \\
(n=17 \text { eyes })\end{array}$ & $\begin{array}{l}\geq 5 \\
(n=53 \text { eyes })\end{array}$ \\
\hline $\begin{array}{l}\text { Extranasal stimulation: Mean (SE) }(\mathrm{mm} / \\
5 \mathrm{~min})\end{array}$ & $0.8(0.8)$ & I.5 (I.I) & $-1.1(0.8)$ & $2.9(1.3)$ & $-0.5(1.0)$ \\
\hline $\begin{array}{l}\text { Intranasal stimulation: Mean (SE) }(\mathrm{mm} / \\
5 \mathrm{~min})\end{array}$ & I3.5 (I.6) & $14.3(1.9)$ & II.I (2.5) & I4.8 (2.8) & $12.6(1.8)$ \\
\hline Difference $(95 \% \mathrm{Cl})(\mathrm{mm} / 5 \mathrm{~min})$ & $\begin{array}{l}-12.7 \\
(-15.1,-10.2)\end{array}$ & $\begin{array}{l}-12.8 \\
(-15.8,-9.8)\end{array}$ & $\begin{array}{l}-12.2 \\
(-16.2,-8.2)\end{array}$ & $\begin{array}{l}-11.9 \\
(-16.7,-7.2)\end{array}$ & $\begin{array}{l}-13.1 \\
(-15.8,-10.4)\end{array}$ \\
\hline P-value & $<0.0001$ & $<0.0001$ & 0.007 & 0.004 & $<0.0001$ \\
\hline
\end{tabular}


Table 3 Comparison of the Effect of Extranasal Stimulation and Intranasal Stimulation on Change from Baseline in Schirmer Scores Between Subgroups Defined by Sjögren Syndrome Medication Use and Baseline Schirmer Score

\begin{tabular}{|c|c|c|}
\hline Change from Baseline in Schirmer Test Score & Extranasal Stimulation & Intranasal Stimulation \\
\hline On Sjögren Syndrome Medication ( $\mathrm{n}=52$ eyes): Mean (SE) $\mathrm{mm} / 5 \mathrm{~min}$ & $1.5(1.1)$ & I4.3 (1.9) \\
\hline Not on Sjögren Syndrome Medication ( $n=18$ eyes): Mean (SE) mm/5 min & $-1.1(0.8)$ & II.I (2.5) \\
\hline Difference $(95 \% \mathrm{Cl}) \mathrm{mm} / 5 \mathrm{~min}$ & $2.6(-0.05,5.2)$ & $3.2(-2.9,9.4)$ \\
\hline P-value & 0.054 & 0.30 \\
\hline Baseline Schirmer $<5 \mathrm{~mm} / 5 \mathrm{~min}$ ( $\mathrm{n}=17$ eyes): Mean (SE) $\mathrm{mm} / 5 \mathrm{~min}$ & $2.9(1.3)$ & $14.8(2.8)$ \\
\hline Baseline Schirmer $\geq 5 \mathrm{~mm} / 5 \mathrm{~min}$ ( $\mathrm{n}=53$ eyes): Mean (SE) mm/5 min & $-0.5(1.0)$ & $12.6(1.8)$ \\
\hline Difference $(95 \% \mathrm{Cl}) \mathrm{mm} / 5 \mathrm{~min}$ & $3.4(0.1,6.6)$ & $2.2(-4.4,8.8)$ \\
\hline P-value & 0.04 & 0.51 \\
\hline
\end{tabular}

a significant improvement in participant ocular discomfort scores. ${ }^{6}$ Finally more recently, Farhangi et al found that in 75 dry eye patients, one session of using the ITN increased tear volume increased tear volume and reduced intensities of dryness and ocular pain. ${ }^{15}$

Similar to these previous studies, we found that the intranasal application of the device significantly increased tear production as compared to baseline, with an average increase of $13.5 \mathrm{~mm}$. This increase was not affected by baseline Schirmer value grouping or systemic SS medication usage, which were potential markers of dry eye and SS disease severity. In addition, the ITN was more effective than intranasal cotton swab stimulation and external application of the device. Although both the cotton swabs and ITN device were physically inserted into the nasal cavity, the device was likely more effective because it also utilizes electrical stimulation.

While it seems that the ITN primarily works through stimulating the lacrimal gland and improving aqueous tear deficiency, there is also evidence that the device may also be able to stimulate production of additional components of the tear film. For example, Pondelis et al used infrared (IR) meibography to measure the dimensional changes of the meibomian glands before and after intranasal stimulation using the device. ${ }^{13}$ Following ITN use, the average meibomian gland area and perimeter significantly decreased suggesting that the meibomian glands contracted after stimulation using the device. The authors concluded that meibum secretion is most likely occurring, which in turn provides further support that other parts of the LFU that contribute to the tear film are also being stimulated by the ITN, such as the meibomian glands and conjunctiva. In addition, Gumus et al studied the effect of the ITN on conjunctival goblet cell degranulation. They enrolled 15 subjects ( 5 normal and
10 dry eyes) and found that the ITN can trigger both mucin cell secretion and an increase in tear volume. ${ }^{12}$

Our study had certain limitations. For example, only patients who were able to produce an increased amount of tears with intranasal cotton swab stimulation were enrolled in the study. Therefore, this limits the generalizability of our results to all SS patients. In addition, because this was a pilot study, complete ocular surface exams were not performed. Future studies that include a more detailed evaluation of the ocular surface would be helpful. Finally, this study had a small sample size. However, we were still able to demonstrate that the ITN device caused a significant increase in tear production from baseline.

In summary, we found that the ITN device can effectively stimulate tear production in a subset of SS patients. While our study had a relatively small sample size and a short duration, it is the first report regarding the efficacy of the ITN exclusively in SS patients. Unfortunately, production of the ITN device was recently discontinued. Some of the barriers to its use included clinical contraindications (deviated septum, chronic anticoagulant use, implanted pacemaker), potential discomfort with device use, and cost. Currently, other treatments are being explored to stimulate tears including an intranasal spray. Our results suggest that SS patients who still have some residual lacrimal gland function could potentially benefit from other future therapies that stimulate the LFU and warrant further study.

\section{Acknowledgments}

The abstract of this paper was presented at the Association for Research in Vision and Ophthalmology 2020 Conference as a poster presentation with interim findings. The poster's abstract was published in "Poster Abstracts" in Investigative Ophthalmology \& Visual Science (https:// iovs.arvojournals.org/article.aspx?articleid=2766590). 


\section{Funding}

Allergan donated the intranasal tear neurostimulator devices and tips for this study.

\section{Disclosure}

VYB reports grant support from Bausch \& Lomb (NEI R01 EY026972); a consultant in 2019 for Verily and also reports patents issued EP 3210201 US 10360819 US 10783505. GSY and PH are supported by NIH grant EY001583 for the statistical work for this paper. MMG is a consultant for Lynthera and owns stocks from PRN. The authors report no other conflicts of interest in this work.

\section{References}

1. Maciel G, Crowson CS, Matteson EL, Cornec D. Prevalence of primary Sjogren's syndrome in a us population-based cohort. Arthritis Care Res. 2017;69(10):1612-1616. doi:10.1002/acr.23173

2. Fox RI. Sjogren's syndrome. Lancet. 2005;366(9482):321-331. doi:10.1016/S0140-6736(05)66990-5

3. Kassan SS, Moutsopoulos HM. Clinical manifestations and early diagnosis of Sjogren syndrome. Arch Intern Med. 2004;164 (12):1275-1284. doi:10.1001/archinte.164.12.1275

4. Kruszka P, O’Brian RJ. Diagnosis and management of Sjogren syndrome. Am Fam Physician. 2009;79(6):465-470.

5. Manthrope R, Asmussen K, Oxholm P. Primary Sjogren's syndrome: diagnostic criteria, clinical features, and disease activity. $J$ Rheumatol Suppl. 1997;50:8-11.

6. Pattar GR, Jerkins G, Evans DG, et al. Symptom improvement in dry eye subjects following intranasal tear neurostimulation: results of two studies utilizing a controlled adverse environment. Ocul Surf. 2020;18 (2):249-257. doi:10.1016/j.jtos.2019.09.006
7. Sheppard JD, Torkildsen GL, Geffin JA, et al. Characterization of tear production in subjects with dry eye disease during intranasal tear neurostimulation: results from two pivotal clinical trials. Ocul Surf. 2019;17(1):142-150. doi:10.1016/j.jtos.2018.11.009

8. Gizurarson S. Anatomical and histological factors affecting intranasal drug and vaccine delivery. Curr Drug Deliv. 2012;9(6):566-582. doi:10.2174/156720112803529828

9. Dieckmann G, Fregni F, Hamrah P. Neurostimulation in dry eye disease-past, present, and future. Ocul Surf. 2019;17(1):20-27. doi:10.1016/j.jtos.2018.11.002

10. Friedman NJ, Butron K, Robledo N, et al. A nonrandomized, open-label study to evaluate the effect of nasal stimulation on tear production in subjects with dry eye disease. Clin Ophthalmol. 2016;10:795-804.

11. Cohn GS, Corbett D, Tenen A, et al. Randomized, controlled, double-masked, multicenter, pilot study evaluating safety and efficacy of intranasal neurostimulation for dry eye disease. Invest Ophthalmol Vis Sci. 2019;60(1):147-153. doi:10.1167/iovs.18-23984

12. Gumus K, Schuetzle KL, Pflugfelder SC. Randomized controlled crossover trial comparing the impact of sham or intranasal tear neurostimulation on conjunctival goblet cell degranulation. Am J Ophthalmol. 2017;177:159-168. doi:10.1016/j.ajo.2017.03.002

13. Pondelis N, Dieckmann GM, Jamali A, et al. Infrared meibography allows detection of dimensional changes in meibomian glands following intranasal neurostimulation. Ocul Surf. 2020;18(3):511-516. doi:10.1016/j.jtos.2020.03.003

14. Shiboski CH, Shiboski SC, Seror R, et al. 2016 American College of Rheumatology/European League Against Rheumatism classification criteria for primary Sjogren's syndrome: a consensus and data-driven methodology involving three international patient cohorts. Ann Rheum Dis. 2017;76(1):9-16. doi:10.1136/annrheumdis-2016210571

15. Farhangi M, Cheng AM, Baksh B, et al. Effect of non-invasive intranasal neurostimulation on tear volume, dryness and ocular pain. Br J Ophthalmol. 2020;104(9):1310-1316.
Clinical Ophthalmology

\section{Publish your work in this journal}

Clinical Ophthalmology is an international, peer-reviewed journal covering all subspecialties within ophthalmology. Key topics include: Optometry; Visual science; Pharmacology and drug therapy in eye diseases; Basic Sciences; Primary and Secondary eye care; Patient Safety and Quality of Care Improvements. This journal is indexed on PubMed

\section{Dovepress}

Central and CAS, and is the official journal of The Society of Clinical Ophthalmology (SCO). The manuscript management system is completely online and includes a very quick and fair peer-review system, which is all easy to use. Visit http://www.dovepress.com/ testimonials.php to read real quotes from published authors. 